\section{Psychiatrische Nebenwirkungen unter 5-alpha- Reduktase-Hemmern}

\author{
Bedenken, wonach die Therapie mit 5-alpha-Reduktase-Inhibitoren ein \\ erhöhtes Suizidrisiko birgt, haben sich in einer kanadischen Studie nicht \\ bestätigt. Völlig harmlos sind die Substanzen aus psychiatrischer Sicht aber \\ offenbar nicht.
}

$\mathrm{M}$ edikamente aus der Klasse der 5-alpha-Reduktase-Inhibitoren (5-ARI), wie sie gegen die benigne Prostatahyperplasie eingesetzt werden, hemmen die Umwandlung von Steroidvorläufern in aktive Hormone. Psychische Effekte dieser Substanzen sind aus mehreren Gründen plausibel. Erstens ist die 5-alpha-Reduktase an der Produktion von Neuropeptiden beteiligt. Zweitens modulieren Testosteron und Dihydrotestosteron die neuroendokrine Stressantwort und sind invers mit Depressionsparametern korreliert. Die Reduktase hat drittens Anteil an der Synthese von Allopregnanolon, das bei Männern mit Depressionen in niedrigerer Konzentration vorliegt. Und viertens findet man bei Männern mit klinischen Depressionen geringere Konzen- trationen von 5-alpha-Reduktase Typ I im präfrontalen Kortex.

Suizide, selbstschädigendes Verhalten und Depressionen sind ein wiederkehrendes Thema im Zusammenhang mit der 5-ARI-Therapie. Ein Ärzteteam um den Urologen Blayne Welk von der Western University in London, Ontario/Kanada, hat diese Verbindungen in einer großangelegten retrospektiven Kohortenstudie untersucht. Ausgewertet wurden, nach Begleitkrankheiten, Arzneigebrauch und Nutzung des Gesundheitssystems 1:1 gematcht, die Daten von mehr als 186.000 Männer im Alter von 66 oder darüber. Der analysierte Zeitraum umfasste die Jahre 2003 bis 2013. Ein statistisch signifikanter Zusammenhang der 5-ARI-Einnahme mit dem Su- izidrisiko war nicht festzustellen. Die Häufigkeit selbstschädigenden Verhaltens war indessen erhöht, und zwar um $88 \%$, wenn auch nur in den ersten 18 Monaten nach Beginn der Medikation. Um 94 \% gesteigert war auch das Risiko, Depressionen zu entwickeln. Nach 18 Monaten schwächte es sich auf $22 \%$ ab, blieb danach aber bestehen. Absolut betrachtet bedeutete das einen Anstieg der Eigenschädigungen von 53,6 auf 70,7 Ereignisse und der Depressionen von 530,1 auf 766,6 Ereignisse je 100.000 Personenjahre. Es spielte dabei keine Rolle, um welchen Typ von 5-ARI es sich handelte. Welk et al. halten es für angebracht, die Medikation unter solchen Umständen abzubrechen.

Fazit: Angesichts des insgesamt geringen absoluten Risikos sollten die Ergebnisse nach Ansicht von Welk und Kollegen keinen Arzt davon abhalten, geeigneten Patienten diese Mittel zu verschreiben.

Dr. Robert Bublak

Welk B et al. Association of Suicidality and Depression With 5a-Reductase Inhibitors. JAMA Intern Med. 2017; doi: 10.1001/jamainternmed.2017.0089.

\title{
Prognoseschwelle für Nierenzellkrebs
}

\section{US-Urologen haben untersucht, wie sich verschiedene Tumorgrößen von nicht metastasierten Nierenzellkarzinomen auf die Prognose auswirken.}

\footnotetext{
n ihrem Artikel in der Fachzeitschrift ,Urology" geben Bimal Bhindi et al. zunächst einen Überblick über die Veränderung der Definition des Schwellenwerts für T1-Nierenzellkarzinome: 1987 verlief die Trennlinie zwischen T1- und T2-Tumoren bei $2,5 \mathrm{~cm}$. Zehn Jahre später wurde die Schwelle auf $7,0 \mathrm{~cm}$ gehoben. $2002 \mathrm{kam}$ eine bei $4,0 \mathrm{~cm}$ verortete Unterscheidung zwischen T1a- und T1bTumoren hinzu. 2010 schließlich führte man eine Trennung von T2a- und T2bTumoren bei $10 \mathrm{~cm}$ ein. Die gegenwärtige Kategorisierung sei nützlich, um Prognosen zuzuordnen und die Konsistenz zwischen Studien zu sichern. Doch sei sie nicht direkt mit Schwellenwerten für das klinische Management verbunden, meinen Bhindi und Kollegen.
}

Um sich einen Eindruck von der Aussagekraft verschiedener Grenzwerte des (jeweils größten) Tumordurchmessers von Nierenzellkarzinomen zu verschaffen, haben Bhindi und Kollegen die prospektiv erhobenen Daten des Nephrektomieregisters der Mayo-Klinik analysiert. In die Berechnungen flossen die Angaben zu 3.304 Patienten im durchschnittlichen Alter von 63 Jahren ein, die zwischen 1970 und 2010 aufgrund eines Nierenzellkarzinoms der Kategorie pT1/2, pNX/0, M0 radikal oder partiell nephrektomiert worden waren. Die mediane Nachbeobachtungszeit betrug 9,9 Jahre.

536 Patienten entwickelten eine Tumorprogression, 354 starben am Nierenkrebs. Für Karzinome bis maximal $3 \mathrm{~cm}$ Durchmesser beliefen sich die Raten des progressionsfreien sowie krebsspezifischen 10-Jahres-Überlebens auf 93-95\% sowie 97-99\%. Jenseits dieser Schwelle nahmen die Quoten ab, bei Tumoren mit einem Durchmesser von 3-4cm lagen die Anteile bei $91 \%$ sowie $95 \%$. Mit zunehmender Tumorgröße sanken die Raten. Prognostische Differenzierungsschwellen mit Blick auf Progression und Mortalität waren nicht auszumachen.

Fazit: „Nierenzellkarzinome bis zu einer Größe von $3 \mathrm{~cm}$ haben eine günstige Prognose", folgern Bhindi et al. Danach stiegen das Progressionsrisiko und die Mortalität graduell mit dem Tumordurchmesser - ohne einen biologisch überzeugenden Grund, einen Schwellenwert gegenüber einem anderen zu bevorzugen. In diesem Fall sollte man in Betracht ziehen, die Schwellen gemäß der klinischen Relevanz festzusetzen. Dr. Robert Bublak

Bhindi B et al. Are We Using the Best Tumor Size Cut-Points for Renal Cell Carcinoma Staging? Urology. 2017; doi: 10.1016/j.urology.2017.04.010. 